\title{
Heterogeneity Measurement Based on Distance Measure for Polarimetric SAR Data
}

\author{
X.L. Xing, Q.H. Chen, X.G. Liu * \\ Faculty of Information Engineering, China University of Geosciences (Wuhan), Wuhan, China; \\ Email address: cug_xxl@163.com; cugcqh@163.com; liuxg318@hotmail.com
}

\begin{abstract}
KEY WORDS: PolSAR Image, Scene Heterogeneity, Distance Measure, Pauli Basis, Polarimetric Information, Textural Features ABSTRACT:
\end{abstract}

To effectively test the scene heterogeneity for polarimetric synthetic aperture radar (PolSAR) data, in this paper, the distance measure is introduced by utilizing the similarity between the sample and pixels. Moreover, given the influence of the distribution and modeling texture, the $\mathrm{K}$ distance measure is deduced according to the Wishart distance measure. Specifically, the average of the pixels in the local window replaces the class center coherency or covariance matrix. The Wishart and $\mathrm{K}$ distance measure are calculated between the average matrix and the pixels. Then, the ratio of the standard deviation to the mean is established for the Wishart and $\mathrm{K}$ distance measure, and the two features are defined and applied to reflect the complexity of the scene. The proposed heterogeneity measure is proceeded by integrating the two features using the Pauli basis. The experiments conducted on the single-look and multilook PolSAR data demonstrate the effectiveness of the proposed method for the detection of the scene heterogeneity.

\section{INTRODUCTION}

Polarimetric SAR systems detect and acquire information by radar echoes of various combinations of transmitting and receiving polarizations from scattering media, and the images have been widely accepted as an indispensable method for earth monitoring (Lee and Pottier. 2009). While the complexity of polarimetric SAR data and the diversity of target scattering complicates the image interpretation and analysis (D'Hondt et al., 2013). Then, the image processing is the emphasis for the application of polarimetric SAR data, such as filtering, decomposition and classification, et al. Simultaneously, because of the ability to objectively and quantitatively describe the difference of the scenes, many adaptive filters have been developed by utilizing the heterogeneity to enhance the performances of speckle filtering (Xing et al.,2017, Lang et al.,2015). Moreover, the heterogeneity could provide useful information for PolSAR image segmentation and classification by reflecting the statistical and spectral differences(Chen et al.,2017). The heterogeneity features were extracted to analyze the saliency of local regions for automatic ship detection(Huang et al,2015), and the coherency matrix estimation employs the heterogeneity coefficient to distinguish the homogeneous and heterogeneous components (Yang et al., 2016). Therefore, the scene heterogeneity plays an important role in PolSAR or SAR information extraction and interpretation.

The coefficient of variation (CV, Lopes et al., 1990) has been recognized as a classical and efficient heterogeneity measure by calculating the ratio of the standard deviation to the mean intensity. In homogeneous scenes, the CV is constant and only related to the number of looks, and the CV is larger with the increase of scenes complexity. Additionally, with its simple calculation, the $\mathrm{CV}$ has been widely applied to detect the presence of texture (Zhong et al., 2011, Yu and Acton, 2002, Dellepiane and Angiati, 2014). Similarly, the ratio of the arithmetic to the geometric mean (A/G) was developed to test SAR image homogeneity as a general index, which showed a good performance for detection of random and structural heterogeneity (Beauchemin et al., 1996). The information-theoretic heterogeneity measurement derived from Shannon's information theory was able to capture subtle variations but with higher computational complexity (Aiazzi et al., 2004). However, these heterogeneity measures proposed were based on the SAR image, which could not make full use of the polarimetric information and affect the test results for PolSAR data.

The Wishart distance measure verifies the similarity between pixels and the class center coherency or covariance matrix (Lee et al., 1994), and the larger distances are closer to the higher heterogeneity region. The difference of the distances is able to reflect the presence of texture in the test area. Thus, this paper develops a heterogeneity measurement for PolSAR image according to the distance measure (DM). The class center coherency or covariance matrix is estimated by using the average of the pixels in the local window. Then, the distances between the pixels and the center matrix are calculated. The complexity of the scene is tested according to the ratio of the standard deviation to the mean of the distances, and the heterogeneity feature is formed. Considering the impact of the distribution and obtaining accurate heterogeneity feature, the $\mathrm{K}$ distance measure is deduced. Subsequently, the heterogeneity measurement is performed by weighing the Wishart and $\mathrm{K}$ heterogeneity features based on the Pauli basis.

Section 2 explains about the proposed heterogeneity measurement approach in detail. The experimental results are presented and discussed in Section 3, and the conclusion is given in Section 4.

\section{PROPOSED METHOD}

\subsection{The Wishart and $\mathrm{K}$ distance measure}

In the monostatic backscattering case, the PolSAR data with horizontal and vertical linear polarizations can be expressed by the scattering matrix, and the scattering matrix of a polarimetric scattering target is represented as (Lee and Pottier. 2009)

$$
\boldsymbol{S}=\left[\begin{array}{ll}
S_{H H} & S_{H V} \\
S_{V H} & S_{V V}
\end{array}\right]
$$

where $S_{H V}$ stands for the scattering coefficient of horizontal $H$ transmitting and vertical $V$ receiving polarizations, and the similar definitions are also suitable for the other elements. For a reciprocal target matrix, the scattering matrix is symmetrical, and the Pauli basis vector $\boldsymbol{k}$ for PolSAR can be expressed as:

*Corresponding author 


$$
\boldsymbol{k}=\frac{1}{\sqrt{2}}\left[S_{H H}+S_{V V} S_{H H}-S_{V V} 2 S_{H V}\right]^{t}
$$

where the superscript $t$ is the transpose operator. In the multilook case, the L-look coherence matrix can be represented as

$$
T=\left\langle\boldsymbol{k} \boldsymbol{k}^{H}\right\rangle=\frac{1}{L} \sum_{i=1}^{L} \boldsymbol{k}(i) \boldsymbol{k}(i)^{* t}
$$

where $\langle\cdot\rangle$ is the ensemble average, $* t$ is the complex conjugate transpose, and $L$ is the number of looks. The coherency matrix $T$ can be described by the Wishart distribution, and the probability density function (PDF) is given by

$$
p_{w}^{(n)}(\boldsymbol{T})=\frac{|\boldsymbol{T}|^{L-q} \exp \left[-\operatorname{tr}\left(\boldsymbol{\Sigma}^{-1} \boldsymbol{T}\right)\right]}{K(L, q)|\boldsymbol{\Sigma}|^{L}}
$$

where $K(L, q)=\pi^{3} \Gamma(L) \ldots \Gamma(L-q+1), q$ is the dimension of matrix $\boldsymbol{T}, \operatorname{tr}(\cdot)$ denotes the trace of a matrix, the covariance matrix $\boldsymbol{\Sigma}$ is the mathematical expectation of $\boldsymbol{T}, \boldsymbol{\Sigma}=E\left[\boldsymbol{k} \boldsymbol{k}^{* t}\right]$. Based on the Wishart distribution and PDF, the maximum likelihood distance measures were derived and applied for segment (Alonso-Gonzalez et al.,2012) and classification (Lee et al.,1999). The distance measure is expressed as follows

$$
\begin{gathered}
d_{w}(\boldsymbol{T}, \boldsymbol{Z})=L \log (|\boldsymbol{Z}|)+\operatorname{tr}\left(\boldsymbol{Z}^{-1} \boldsymbol{T}\right)-(L-q) \log |\boldsymbol{T}| \\
+\log (K(L, q))
\end{gathered}
$$

In this paper, the class center matrix is replaced by the local mean to test the presence of the texture, and $\boldsymbol{Z}$ denotes the average of the local window.

The Wishart distribution is based on the assumption that speckle is fully developed and have a zero-mean circular complex Gaussian process, which is suitable to describe the homogeneous region. In addition, the K-distribution has been proved to be useful in modeling the coherency matrix for heterogeneous areas with rich texture information, and the PDF is expressed as follows

$$
p_{k}(\boldsymbol{T})=\frac{2|\boldsymbol{T}|^{L-q}(L \alpha)^{(\alpha+q L) / 2}}{K(L, q) \Gamma(\alpha)} \cdot \frac{K_{\alpha-q L}\left(2 \sqrt{\operatorname{tr}\left(\boldsymbol{\Sigma}^{-1} \boldsymbol{T}\right) L \alpha}\right)}{|\boldsymbol{\Sigma}|^{L} \operatorname{tr}\left(\boldsymbol{\Sigma}^{-1} \boldsymbol{T}\right)^{(q L-\alpha) / 2}}
$$

where $K_{v}(\cdot)$ is the modified Bessel function of the second kind with order $v . \alpha$ is the shape parameter, and $\Gamma(\cdot)$ is the gamma function. Similarly, the $\mathrm{K}$ distance measure is derived according to the K-distribution PDF and given by

$$
\begin{gathered}
d_{k}(\boldsymbol{T}, \boldsymbol{Z})=\ln (\Gamma(\alpha))+L \ln (|\boldsymbol{Z}|)+\frac{q L-\alpha}{2} \ln \left(\operatorname{tr}\left(\boldsymbol{Z}^{-1} \boldsymbol{T}\right)\right) \\
-(L-q) \ln (2|\boldsymbol{T}|)-\frac{\alpha+q L}{2} \ln (L \alpha) \\
-\ln \left(K_{\alpha-q L}\left(2 \sqrt{\operatorname{tr}\left(\boldsymbol{Z}^{-1} \boldsymbol{T}\right) L \alpha}\right)\right)
\end{gathered}
$$

\subsection{Heterogeneity Measurement Based on Distance Measure}

Based on the above distance measure, for PolSAR images, we can measure the similarity distance between pixels and the local mean. Moreover, the distances are nearly equal with more homogeneous, on the contrary, the difference between the distances increases with the richer texture. Thus, the heterogeneity can be measured according to the distance measure by using the ratio of the standard deviation to the mean.
As the terrain appears more homogeneous with the shape parameter increasing, the $\mathrm{K}$ distance measure goes to infinity. The heterogeneity based on the distance measure utilizing the Pauli basis is expressed as

$$
H=H_{w i}+P_{i} \cdot H_{k i}
$$

Here, subscription $i$ is the pixel position in sliding window. $H_{w i}$ and $H_{k i}$ denote the heterogeneity features calculated from the ratio of the standard deviation to the mean based on the Wishart and $\mathrm{K}$ distance measure, respectively, and which can be given as follows: $H_{w i}=\frac{\operatorname{std}\left(d_{w i}\right)}{\operatorname{mean}\left(d_{w i}\right)}, H_{k i}=\frac{\operatorname{std}\left(d_{k i}\right)}{\operatorname{mean}\left(d_{k i}\right)} . \quad P_{i}$ corresponds to the change of polarimetric information of local areas and calculated based on the three components of the Pauli basis, $P_{i}=\max \left(\frac{\operatorname{std}\left(\text { pauli }_{n}\right)}{{\text { mean }\left(\text { pauli }_{n}\right)}_{1}}\right)$, subscription $n$ is the polarization channels of the Pauli basis.

Specifically, the procedure of the proposed method could be described as follows:

(1) Calculate the average coherency or covariance matrix of the sliding window.

(2) For each pixel of the sliding window, utilize the Wishart and $\mathrm{K}$ distance measure to calculate $d_{w}$ and $d_{k}$, respectively.

(3)According to the ratio of the standard deviation to the mean of $d_{w}$ and $d_{k}$, obtain the Wishart and K heterogeneity features $H_{w i}$ and $H_{k i}$.

(4)Alter the largest component $P_{i}$ to measure the polarimetric information in the sliding window, which is measured by using the three components of the Pauli basis to compute the ratio of the standard deviation to the mean.

(5) Integrate polarimetric information and the Wishart and $\mathrm{K}$ heterogeneity features to measure the scene heterogeneity.

\section{EXPERIMENTAL RESULTS AND ANALYSIS}

For illustration, two real PolSAR images are applied to validate the effectiveness of the proposed approach. The first experimental dataset is selected from the four-look AIRSAR image over San Francisco, including water, forest, point targets and urban areas with a size of $400 \times 400$ pixels. The second employed dataset is a section of the one-look ESAR image of Oberpfaffenhofen, which is $600 \times 600$ pixels in size and composed of different classes with weak and strong point objects, line, and field areas. The corresponding Pauli decomposition images are shown in Figure.1 (a) and (b), respectively.

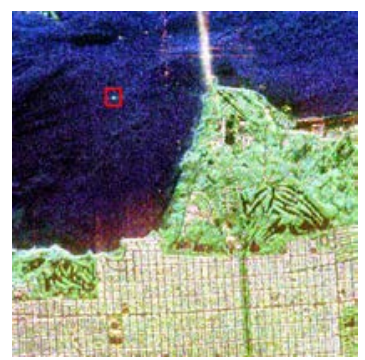

(a)

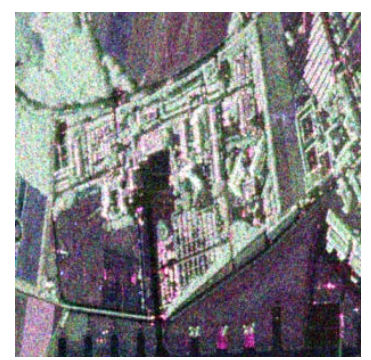

(b)
Figure 1.Original data based on the Pauli decomposition:

(a) Original data of AIRSAR; and (b) Original data of ESAR

The experiments were performed by utilizing different methods, including (a) the CV; (b) the A/G; (c) the shape parameter; and (d) the proposed DM. The CV was the most commonly used method to measure heterogeneity, for PolSAR data, the span image was applied to calculate the ratio of the standard 
deviation to the mean. Similarly, the A/G was performed based on the span image. The shape parameter was an important parameter of $\mathrm{K}$ distribution and could determine the degree of heterogeneity of the synthetic data (Khan and Guida,2014).

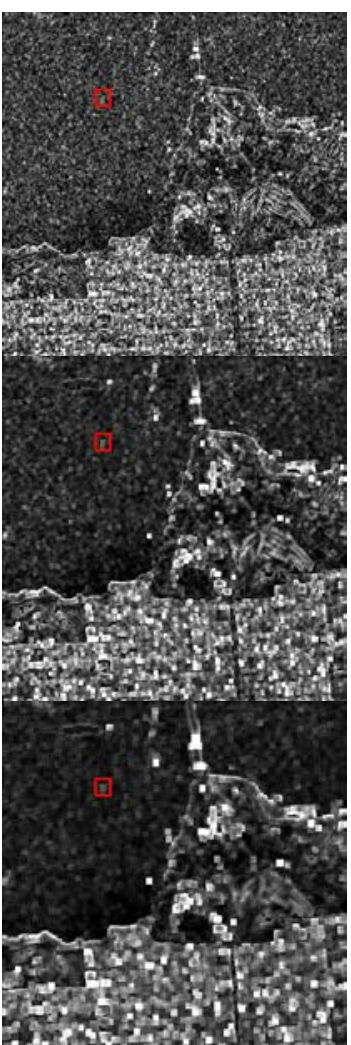

(a)

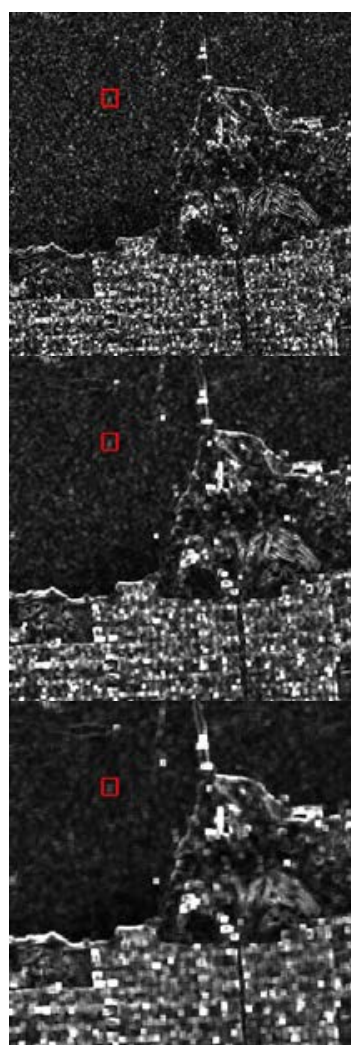

(b)
Simultaneously, given the influence of the sliding window sizes, the experiments were conducted based on the heterogeneity methods with ranging from $3 \times 3$ to $7 \times 7$.
Figure 2. Comparison of the heterogeneity feature on AIRSAR data. From top to bottom are the heterogeneity

images with ranging from $3 \times 3$ to $7 \times 7$. (a) $\mathrm{CV}$; (b) A/G; (c) shape parameter; (d) the proposed DM.
Figure 2 displays the experimental results on AIRSAR data. As can be seen, most features could be measured by different methods. While, the points in the red boxes are smeared in the $\mathrm{CV}$ and $\mathrm{A} / \mathrm{G}$ maps presented on columns (a) and (b). Obviously, the heterogeneity maps of the shape parameter and the proposed DM are detailed especially on bridge areas on columns (c) and (d). Moreover, as can be seen, the heterogeneity maps of the shape parameter appear sharp, and the proposed DM maps show larger contrast with the increase of window size. For the ESAR image, the heterogeneity maps calculated are shown in Figure 3. It is evident that the noisiness of the heterogeneity map of the shape parameter makes the features blurred with window size $3 \times 3$. Correspondingly, other heterogeneity maps are more detailed. Additionally, the textured forested area emerges better in the three maps of the proposed DM. These maps confirm that the proposed method is a powerful heterogeneity measurement.

Based on the above analysis, the heterogeneity features are affected by speckles with the smaller size of calculation windows, while the details could be clearly captured. In addition, the effect of speckle noise is reduced by expanding the window size, while the fine details are smoothed and spread. Hence, the size of the sliding window could be adjusted depending on the application.

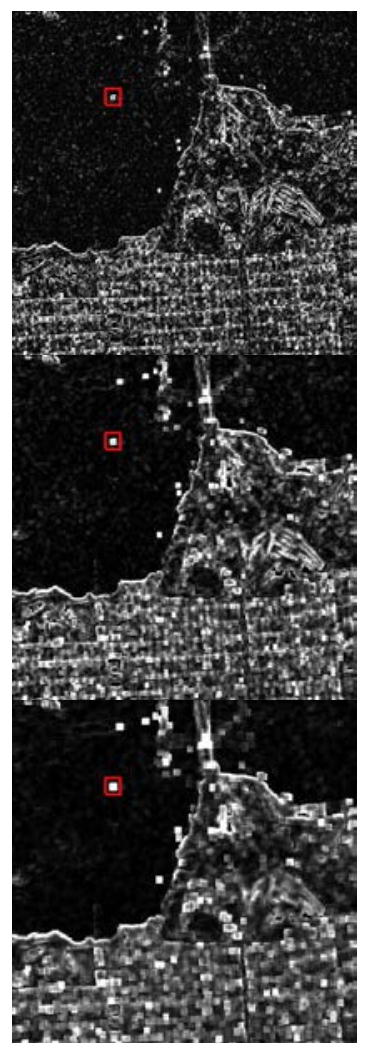

(c)

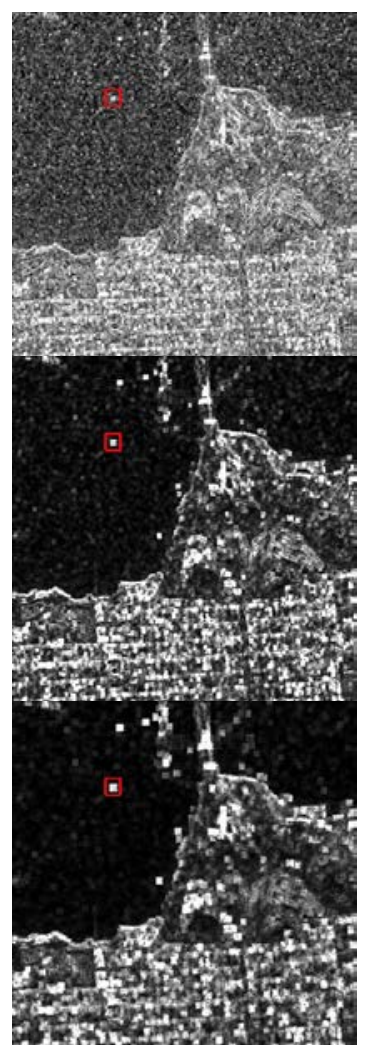

(d)

\section{CONCLUDING}

In this study, the distance measure is introduced to describe the spatial heterogeneity for PolSAR data. The ratio of the standard deviation to the mean of the Wishart and $\mathrm{K}$ distance measure is calculated, and the two features are established. The novel heterogeneity measurement is performed by combining the two features according to the Pauli basis. This approach makes full advantage of the polarimetric information, which is more suitable for PolSAR data. In addition, the combination of the Wishart and $\mathrm{K}$ distance measure is capable of enhancing the stability to adapt single-look and multilook PolSAR images. In particular, the effectiveness of the proposed DM in the smaller window size indicates promise for the development of the PolSAR image processing, including the filters and segmentation, et al. The experiments of different methods with AIRSAR and ESAR PolSAR data demonstrate the performance of the proposed heterogeneity measurement. 


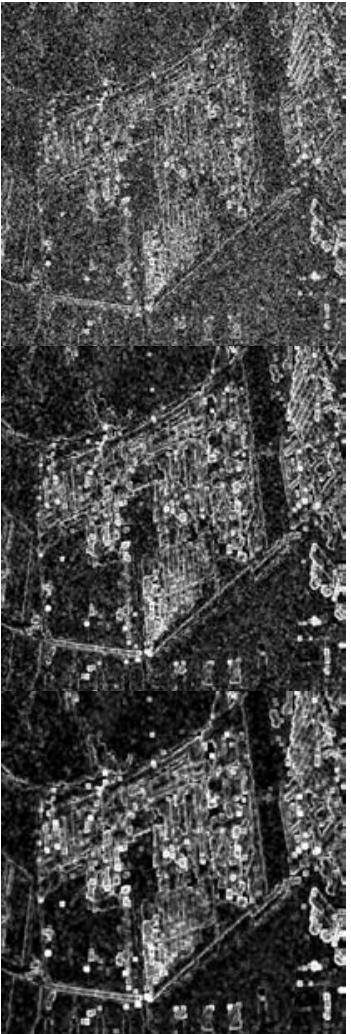

(a)

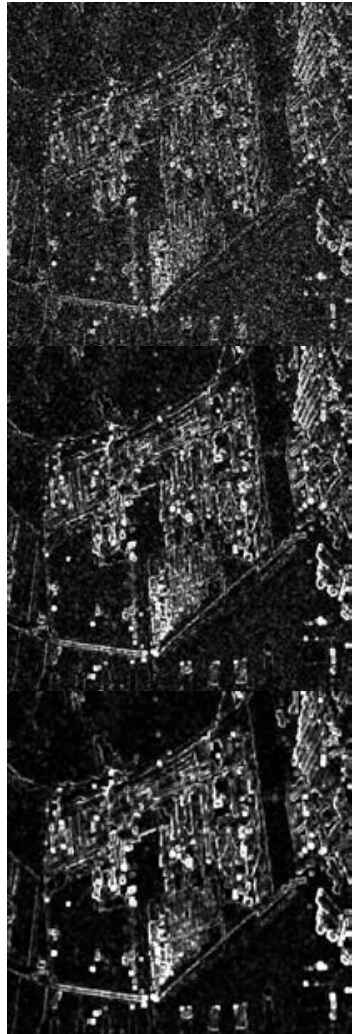

(b)

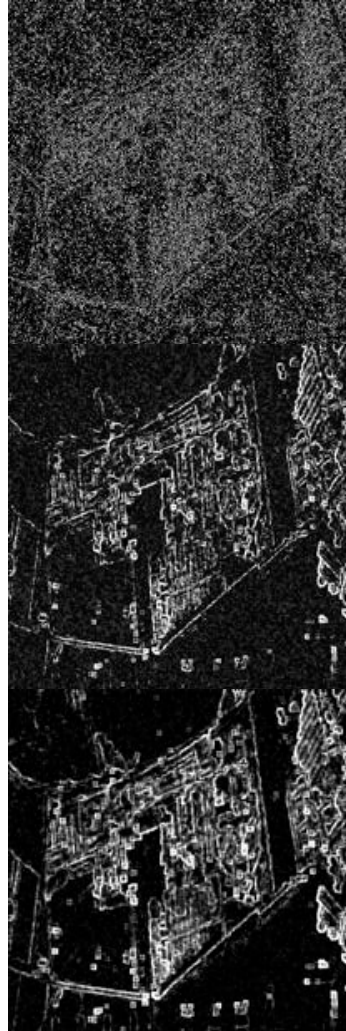

(c)

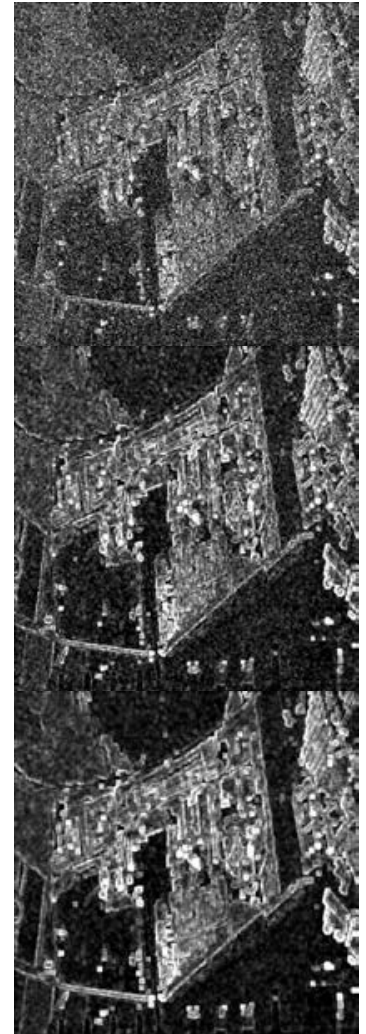

(d)

Figure 3. Comparison of the heterogeneity feature on ESAR data. From top to bottom are the heterogeneity images with ranging from $3 \times 3$ to $7 \times 7$. (a) $\mathrm{CV}$;(b)A/G;(c)shape parameter;(d)the proposed DM.

\section{Acknowledgements}

This work is supported in part by the National Natural Science Foundation of China under grant no. 41471355, 41771467.

\section{References}

Alonso-Gonzalez, A.; Lopez-Martinez, C.; Salembier, P. 2012. Filtering and segmentation of polarimetric SAR data based on binary partition Trees. Geoscience and Remote Sensing, IEEE Transactions on, 50(2), 593-605.

Aiazzi, B., Alparone, L., and Baronti, S. 2004. Information-Theoretic Heterogeneity Measurement for SAR Imagery. Geoscience and Remote Sensing, IEEE Transactions on,43(3): pp. 619-624.

Beauchemin,M., Thomson,K. P.-B., and Edwards, G. 1996.The Ratio of the Arithmetic to the Geometric Mean: A First-Order Statistical Test for Multilook SAR Image Homogeneity. Geoscience and Remote Sensing, IEEE Transactions on, 34(2): pp. 604-606. Chen,Q.-H., Li, L.-L., Xu, Q., Yang S., Shi, X.-G., Liu, X.-G. Multi-Feature Segmentation for High-Resolution Polarimetric SAR Data Based on Fractal Net Evolution Approach. Remote Sensing . 2017, 9, 570.

D’Hondt, O., Guillaso, S., and Hellwich, O. 2013. Iterative Bilateral Filtering of Polarimetric SAR Data. Selected Topics in Applied Earth Observations and Remote Sensing, IEEE Journal of, 6(3): pp.
1628-1639.

Dellepiane, S.-G.,and Angiati, E. Quality assessment of despeckled SAR images. Selected Topics in Applied Earth Observations and Remote Sensing, IEEE Journal of 2014, 7(2):pp. 691-707.

Huang, X.-J., Yang, W., Zhang, H.-J., Xia, G.-Song. 2015. Automatic Ship Detection in SAR Images Using Multi-Scale Heterogeneities and an A Contrario Decision. Remote Sensing, 7:7695-7711.

Khan, S. and Guida, R. 2014.On fractional moments of multilook polarimetric whitening filter for polarimetric SAR data. Geoscience and Remote Sensing, IEEE Transactions on, 52(6), pp. 3502-3512.

Lee, J.-S.; Grunes, M.R.; Ainsworth, T.L.; Du, L.; Schuler, D.L.; Cloude, S.R. 1999.Unsupervised classification using polarimetric decomposition and the complex Wishart classifier. Geoscience and Remote Sensing, IEEE Transactions on, 37(5), 2249-2258.

Lee, J.-S., Grunes, M.-R. and Kwok, R. 1994. Classification of multi-look polarimetric SAR imagery based on complex Wishart distribution, International Journal of Remote Sensing, 15(11), pp.2299-2311.

Lee, J.-S. and Pottier, E. 2009. Polarimetric radar imaging: from basics to applications. CRC press.

Lopes,A., Nezry,E., Touzi,R., and Laur,H.1990. Adaptive Speckle Filters and Scene Heterogeneity. Geoscience and Remote Sensing, IEEE Transactions on, 28(6): pp. 992-1000.

Lang, F.-K, Yang, J, and Li, D.-R. 2015. Adaptive Window Polarimetric SAR Image Speckle Filtering Based on a Homogeneity Measurement. Geoscience 
and Remote Sensing, IEEE Transactions on, 53(10): 5435-5446.

Xing, X.-L, Chen, Q.-H, Yang, S., Liu, X.-G. 2017. Feature-Based Nonlocal Polarimetric SAR Filtering. Remote Sensing , 9, 1043.

Yu,Y.-J.,and Acton,S.-T. 2002. Speckle Reducing Anisotropic Diffusion. Image Processing, IEEE Transactions on 11(11), pp. 1260-1270.

Yang, S., Chen, Q.-H, Yuan, X.-H., Liu, X.-G. 2016. Adaptive CoherencyMatrix Estimation for Polarimetric SAR Imagery Based on Local Heterogeneity Coefficients. Geoscience and Remote Sensing, IEEE Transactions on, 54(11): 6732-6745.

Zhong,H. , Li,Y.-Wei., and Jiao,L.-C.2011. SAR Image Despeckling Using Bayesian Nonlocal Means. IEEE Geoscience and Remote Sensing Letters 8 (4): pp. 809-813. 